\title{
Heat Kernels of Multiplicative Perturbations: Hölder Estimates and Gaussian Lower Bounds
}

\section{El Maati Ouhabaz}

ABSTRACT. We study in an abstract setting heat kernels of multiplicative perturbations $b A$ where $b$ is a bounded measurable function. Our aim is to show that Hölder estimates and Gaussian lower bounds carry over from the heat kernel of $A$ to the heat kernel of $b A$. In preparing these results we show that Hölder continuity estimates of heat kernels can be characterized in terms of Gagliardo-Nirenberg type inequalities.

1. Introduction. The aim of this note is to study Hölder continuity and Gaussian lower bounds for bounded multiplicative perturbations. We consider the following general framework: $X$ is a topological space equipped with a metric $d$ and a $\sigma$-finite measure $\mu$, and consider an operator $A$ which generates an analytic semigroup $e^{-t A}$ in $L^{p}(X)$. We assume that $e^{-t A}$ has a kernel $p_{t}(x, y)$. We are interested in heat kernels $k_{t}(x, y)$ of bounded multiplicative perturbations $b A\left(b \in L^{\infty}(X)\right.$ and $\left.\Re b(x) \geq \delta>0\right)$. Our main interest is to obtain Gaussian upper and lower bounds and Hölder continuity estimates for $k_{t}(x, y)$ assuming that one knows these properties for $p_{t}(x, y)$. For Gaussian upper bounds, this has been done in $[\mathrm{DO}]$. It is shown there that if

$$
0 \leq p_{t}(x, y) \leq C t^{-D / 2} e^{-c\left(d(x, y)^{2}\right) / t}, \quad t>0,
$$

then the kernel $k_{t}(x, y)$ of $e^{-t b A}$ satisfies

$$
\left|k_{t}(x, y)\right| \leq C^{\prime} t^{-D / 2} e^{-c^{\prime}\left(d(x, y)^{2}\right) / t}, \quad t>0 .
$$

This extended a result of McIntosh and Nahmod [McN] who studied the case where $A$ is the Laplacian on $L^{2}\left(\mathbb{R}^{D}\right)$. They showed that $k_{t}(x, y)$ has a polynomial decay in $|x-y|$ and mentioned that one can improve it to exponential decay by making a good control of the constants involved in their estimates. In the 
present paper we show Hölder continuity and Gaussian lower bounds for $k_{t}(x, y)$. Concerning Hölder continuity, we show that if

$$
\left|p_{t}(x, y)-p_{t}\left(x^{\prime}, y\right)\right| \leq C t^{-D / 2-\eta / 2} d\left(x, x^{\prime}\right)^{\eta}, \quad t>0, x, x^{\prime}, y \in X,
$$

then the heat kernel $k_{t}(x, y)$ of $B A$ satisfies the same estimate whenever the operator $B$ and its inverse $B^{-1}$ are bounded on $L^{p}(X)$ for some $p$ (see Theorem $2)$. Such Hölder estimates are of great interest in the study of evolution equations. In particular, they allow to obtain Gaussian lower bounds on the heat kernel which are important for obtaining Harnack inequalities. The latter being a power tool in the study of parabolic equations. Note that for second order elliptic operators in divergence form, there are several relations between Harnack inequalities, upper and lower Gaussian bounds of the heat kernel and Poincaré inequalities (see [Sc2] and the references there). In our situation, $A$ is an abstract operator and we ask whether it is possible to obtain a characterization of Hölder continuity of $p_{t}(x, y)$ in terms of $A$. We give such a characterization in terms of Gagliardo-Nirenberg type inequalities

$$
\operatorname{Sup}_{x \neq x^{\prime}} \frac{\left|f(x)-f\left(x^{\prime}\right)\right|}{d\left(x, x^{\prime}\right)^{\eta / p}} \leq C\|f\|_{p}^{1-(1 / \beta)(\eta / p+D / p)}\left\|A_{p}^{\beta / 2} f\right\|_{p}^{(1 / \beta)(\eta / p+D / p)}
$$

for all $\beta, p$ such that $\beta>\eta / p+D / p$ (see Theorem 1 for the precise statement).

This criterion can be used easily when dealing with perturbations of $A$ and in particular, for bounded multiplicative perturbations. In fact, if $B, B^{-1} \in$ $\mathcal{L}\left(L^{p}(X)\right)$ then $\|A f\|_{p} \leq\left\|B^{-1}\right\| \cdot\|B A f\|_{p}$, which implies that $B A$ satisfies the above inequality (with $\beta=2$ ). We then conclude that the kernel $k_{t}(x, y)$ of $B A$ satisfies

$$
\left|k_{t}(x, y)-k_{t}\left(x^{\prime}, y\right)\right| \leq C t^{-D / 2-\eta_{0} / 2} d\left(x, x^{\prime}\right)^{\eta_{0}}
$$

for some positive $\eta_{0}$.

The idea of characterizing Hölder estimates in terms of Gagliardo-Nirenberg type inequalities has its roots in the work of Coulhon [Co1]. In that paper it was shown that the $L^{p}-L^{\infty}$ estimate

$$
\left\|e^{-t A} f\right\|_{\infty} \leq C t^{-D /(2 p)}\|f\|_{p}
$$

is equivalent to the Gagliardo-Nirenberg inequality

$$
\|f\|_{\infty} \leq C\|f\|_{p}^{1-D /(\beta p)}\left\|A^{\beta / 2} f\right\|_{p}^{D /(\beta p)}, \quad \text { for } \beta p>D .
$$

We will follow the same ideas of the proof given in [Co1] and show that if we know the Hölder estimate then we may replace the $L^{\infty}$-norm in the last inequality by $\operatorname{Sup}_{x \neq x^{\prime}}\left|f(x)-f\left(x^{\prime}\right)\right| / d\left(x, x^{\prime}\right)^{\eta / p}$.

Another aim of this paper is Gaussian lower bounds. It is natural to ask whether Gaussian lower bounds carry over from the kernel $p_{t}(x, y)$ to the heat kernel $k_{t}(x, y)$ of $b A\left(b \in L^{\infty}(X)\right.$ with $\left.b(x) \geq \delta>0\right)$. In view of the result 
given in [DO] for Gaussian upper bounds it seems likely that one could answer positively this question but we have not succeeded in doing this. However, we can answer positively the question if we assume that $A$ is self-adjoint and $p_{t}(x, y)$ is Hölder continuous. In this case, we are able to show a uniform lower bound

$$
k_{t}(x, x) \geq C t^{-D / 2}, \quad t>0
$$

and by the previous results we know that $k_{t}(x, y)$ is also Hölder continuous. Now, it is classical that these two properties imply a Gaussian lower bound (see Davies [Da1, Chap. 3] or Saloff-Coste [Sc1]).

There are several classes of operators $A$ which satisfy these assumptions.

For example, second-order self-adjoint elliptic operators with real coefficients on $\mathbb{R}^{D}$ or on regular domains ([Da1, Chap. 3], [Fr], $[\mathrm{Au}]$ ). Other examples are sub-Laplacians on Lie groups of polynomial volume growth ([Sc1], [VSC]) and Laplace-Beltrami operators on manifolds with non-negative Ricci curvature $([\mathrm{LY}])$.

Remark. Throughout this paper we often denote by $C$ all inessential constants. The value of $C$ may change from line to line.

Acknowledgements. I wish to express my thanks to Thierry Coulhon and Xuan Thinh Duong for reading a first draft of this paper and for their comments and help.

\section{Hölder continuity and Gagliardo-Nirenberg type inequalities.} As above, the triple $(X, d, \mu)$ denotes a topological metric space equipped with a measure $\mu$. We denote by $C^{\eta}(X), 0<\eta<1$, the space of scalar-valued functions $f$ defined on $X$ which are bounded and satisfy

$$
\operatorname{Sup}_{x \neq x^{\prime}} \frac{\left|f(x)-f\left(x^{\prime}\right)\right|}{d\left(x, x^{\prime}\right)^{\eta}}<\infty
$$

The norm of the space $C^{\eta}(X)$ is given by

$$
\|f\|_{\infty}+\operatorname{Sup}_{x \neq x^{\prime}} \frac{\left|f(x)-f\left(x^{\prime}\right)\right|}{d\left(x, x^{\prime}\right)^{\eta}} .
$$

Let $-A$ be the generator of a bounded holomorphic semigroup $e^{-t A}$ on $L^{2}(X):=$ $L^{2}(X, \mu)$. We assume that the semigroup $e^{-t A}$ is uniformly bounded on $L^{p}(X)$, $1 \leq p \leq \infty$. We keep the same notation $e^{-t A}$ to denote the induced bounded analytic semigroup on $L^{p}(X), 1<p<\infty$, but write $-A_{p}$ to indicate the corresponding generator on $L^{p}(X)\left(A_{2}=A\right)$. Suppose now that the semigroup $e^{-t A}$ has a "heat" kernel $p_{t}(x, y)$. We consider the following Hölder continuity 
assumption on $p_{t}(x, y)$

$$
\begin{aligned}
\left|p_{t}(x, y)-p_{t}\left(x^{\prime}, y\right)\right| & \leq C t^{-D / 2-\eta / 2} d\left(x, x^{\prime}\right)^{\eta}, \\
& \text { for all } t>0, x, x^{\prime} \in X \text { and } \mu \text {-a.e. } y \in X
\end{aligned}
$$

where $C, D$, and $\eta \in(0,1)$ are positive constants.

This assumption implies in particular that the solution of the "heat" equation

$$
\left\{\begin{array}{l}
\frac{\partial}{\partial t} u(t, \cdot)=-A u(t, \cdot) \\
u(0, \cdot)=f
\end{array}\right.
$$

is Hölder continuous and satisfies the estimate

$$
\left|u(t, x)-u\left(t, x^{\prime}\right)\right| \leq C d\left(x, x^{\prime}\right)^{\eta} t^{-D / 2-\eta / 2}\|f\|_{1}
$$

for initial data $f \in L^{1}(X) \cap L^{2}(X)$.

As we mentioned in the introduction, the estimate (1) permits also to obtain Gaussian lower bounds and Harnack inequalities from Gaussian upper bounds and on-diagonal lower bounds of the heat kernel.

Our first aim is to characterize (1) in terms of the operator $A$. We search for a characterization which permits to show that Hölder continuity carries over from the heat kernel of $A$ to heat kernels of other perturbations of $A$ and in particular bounded multiplicative perturbations. Before giving the result let us consider the following $L^{1}-L^{\infty}$ estimate of $e^{-t A}$ :

$$
e^{-t A} \text { is bounded from } L^{1}(X) \text { into } L^{\infty}(X) \text { with norm }
$$

$$
\left\|e^{-t A}\right\|_{1, \infty} \leq C t^{-D / 2}, \quad t>0
$$

This kind of estimate is well studied and can be characterized in several ways, for example in terms of Nash, Sobolev or logarithmic Sobolev type inequalities (see [Da1], [VSC] and the references therein).

Another characterization of (2) is given by Theorem 1 of [Co1], which says precisely that the $L^{p}-L^{\infty}$ estimate

$$
\left\|e^{-t A} f\right\|_{\infty} \leq C t^{-D /(2 p)}\|f\|_{p}
$$

is equivalent to the Gagliardo-Nirenberg inequality

$$
\|f\|_{\infty} \leq C\|f\|_{p}^{1-D /(\beta p)}\left\|A^{\beta / 2} f\right\|_{p}^{D /(\beta p)}, \quad \text { for } \beta p>D
$$

In the light of (3) it comes a natural question to ask whether one can also rely the Hölder continuity estimate (1) to a Gagliardo-Nirenberg type inequality by replacing the $L^{\infty}$-norm by the $C^{\eta}$-one. The answer is given in the following result: 
Theorem 1 Suppose that $e^{-t A}$ is uniformly bounded on $L^{p}(X), 1 \leq p \leq \infty$ and bounded analytic on $L^{p}(X), 1<p<\infty$. If (1) holds, then A satisfies the following inequalities:

$$
\operatorname{Sup}_{x \neq x^{\prime}} \frac{\left|f(x)-f\left(x^{\prime}\right)\right|}{d\left(x, x^{\prime}\right)^{\frac{\eta}{p}}} \leq C\|f\|_{p}^{1-(1 / \beta)(\eta / p+D / p)}\left\|A_{p}^{\beta / 2} f\right\|_{p}^{(1 / \beta)(\eta / p+D / p)}
$$

for all $p \in(1, \infty)$ and $\beta$ such that $\beta>\eta / p+D / p$ and all $f \in D\left(A_{p}^{\beta / 2}\right)$.

Conversely, assume that (2) holds. If $A$ satisfies (4) for some $\beta$ and $p>1$ with $\beta>\eta / p+D / p$, then $p_{t}(x, y)$ satisfies (1) with $\eta / p$ in place of $\eta$.

We also have the same conclusions with $p=1$ if we assume that $e^{-t A}$ is bounded analytic on $L^{1}(X)$.

This result implies in particular that if $p_{t}(x, y)$ satisfies both (1) and (2), then $D\left(A_{p}^{\beta / 2}\right) \subset C^{\eta / p}(X)$ for $\beta>\eta / p+D / p$.

We mention that related questions are studied in [Co3] in the setting of graphs and Riemannian manifolds. Geometrical conditions are given there which guaranted the Sobolev inequalities

$$
\left|f(x)-f\left(x^{\prime}\right)\right| \leq C d\left(x, x^{\prime}\right)^{\eta}\|\nabla f\|_{p} .
$$

These estimates are of different nature than (4) because it may happen that the norms $\|\nabla f\|_{p}$ and $\left\|\Delta^{1 / 2} f\right\|_{p}$ are not equivalent. In addition, as we are interested in getting Hölder estimates for heat kernels, then (4) seems to be more convenient because, in general, it is not easy to estimate the $L^{p}$-norm of $\nabla e^{-t \Delta}$. Hence our result can be seen as complementary to those in [Co3, section 4].

Proof. We shall follow the same ideas as in [Co1]. Assume that $A$ satisfies (4) with some $\beta$ and $p>1(\beta>\eta / p+D / p)$. Using (2) and the fact that the semigroup $e^{-t A}$ is bounded analytic on $L^{p}(X)$, it is easy to see that $e^{-t A}$ maps $L^{1}(X)$ into $D\left(A_{p}^{m}\right)$ for all $m \geq 0$. We apply now (4) with $e^{-t A} f, f \in L^{1}(X)$. We have

$$
\begin{aligned}
& \left|e^{-t A} f(x)-e^{-t A} f\left(x^{\prime}\right)\right| \\
& \quad \leq C d\left(x, x^{\prime}\right)^{\eta / p}\left\|e^{-t A} f\right\|_{p}^{1-(1 / \beta)(\eta / p+D / p)}\left\|A_{p}^{\beta / 2} e^{-t A} f\right\|_{p}^{(1 / \beta)(\eta / p+D / p)} .
\end{aligned}
$$

From (2) we have

$$
\left\|e^{-t A} f\right\|_{p} \leq C t^{(-D / 2)(1-1 / p)}\|f\|_{1}
$$

and by analyticity

$$
\left\|A_{p}^{\beta / 2} e^{-t A} f\right\|_{p} \leq C t^{-\beta / 2}\left\|e^{-(t / 2) A} f\right\|_{p} .
$$

Hence

$$
\left|e^{-t A} f(x)-e^{-t A} f\left(x^{\prime}\right)\right| \leq C t^{-D / 2-\eta /(2 p)} d\left(x, x^{\prime}\right)^{\eta / p}\|f\|_{1} .
$$


This clearly implies (1) with $\eta / p$ in place of $\eta$.

The same argument shows that if the semigroup $e^{-t A}$ is bounded analytic on $L^{1}(X)$ then we have the same conclusion with $p=1$.

Conversely, assume that (1) holds. This can be written for a given $x$ and $x^{\prime}$ as

$$
\left\|p_{t}(x, .)-p_{t}\left(x^{\prime}, \cdot\right)\right\|_{\infty} \leq C t^{-D / 2-\eta / 2} d\left(x, x^{\prime}\right)^{\eta} .
$$

The fact that the semigroup $e^{-t A}$ is uniformly bounded on $L^{\infty}(X)$ implies that

$$
\left\|p_{t}(x, \cdot)\right\|_{1} \leq C \text { for all } x \in X, t>0 .
$$

Now the interpolation inequality implies that for all $p \in(1, \infty)$

$$
\left\|p_{t}(x, \cdot)-p_{t}\left(x^{\prime}, \cdot\right)\right\|_{p} \leq C t^{(1-1 / p)(-D / 2-\eta / 2)} d\left(x, x^{\prime}\right)^{\eta(1-1 / p)} .
$$

Let now $f \in L^{p}(X)$ and $t>0$. By Hölder's inequality we have

$$
\begin{aligned}
\left|e^{-t A} f(x)-e^{-t A} f\left(x^{\prime}\right)\right| & =\left|\int_{X}\left(p_{t}(x, y)-p_{t}\left(x^{\prime}, y\right)\right) f(y) d \mu(y)\right| \\
& \leq\left\|p_{t}(x, \cdot)-p_{t}\left(x^{\prime}, \cdot\right)\right\|_{q}\|f\|_{p}
\end{aligned}
$$

where $1 / p+1 / q=1$. Using (6), we obtain the estimate

$$
\left|e^{-t A} f(x)-e^{-t A} f\left(x^{\prime}\right)\right| \leq C d\left(x, x^{\prime}\right)^{\eta / p} t^{-D /(2 p)-\eta /(2 p)}\|f\|_{p} .
$$

We now show (4). Suppose first that $\beta \leq 2$. Let $f \in D\left(A_{p}^{\beta / 2}\right)$ with $\beta>$ $\eta / p+D / p$. Write

$$
f=e^{-t A} f+\int_{0}^{t} e^{-(s / 2) A} A_{p}^{1-\beta / 2} e^{-(s / 2) A} A_{p}^{\beta / 2} f d s .
$$

Using (7), we have

$$
\begin{gathered}
\left|e^{-(s / 2) A} A_{p}^{1-\beta / 2} e^{-(s / 2) A} A_{p}^{\beta / 2} f(x)-e^{-(s / 2) A} A_{p}^{1-\beta / 2} e^{-(s / 2) A} A_{p}^{\beta / 2} f\left(x^{\prime}\right)\right| \\
\leq C d\left(x, x^{\prime}\right)^{\eta / p} s^{-D /(2 p)-\eta /(2 p)}\left\|A_{p}^{1-\beta / 2} e^{-(s / 2) A} A_{p}^{\beta / 2} f\right\|_{p},
\end{gathered}
$$

and by analyticity,

$$
\left\|A_{p}^{1-\beta / 2} e^{-(s / 2) A} A_{p}^{\beta / 2} f\right\|_{p} \leq C s^{\beta / 2-1}\left\|A_{p}^{\beta / 2} f\right\|_{p} .
$$

From (8) and these two estimates we have (for all $x \neq x^{\prime}$ )

$$
\begin{aligned}
\frac{\left|f(x)-f\left(x^{\prime}\right)\right|}{d\left(x, x^{\prime}\right)^{\eta / p}} \leq C & t^{-\eta /(2 p)-D /(2 p)}\|f\|_{p} \\
& +C \int_{0}^{t} s^{\beta / 2-1-\eta /(2 p)-D /(2 p)} d s\left\|A_{p}^{\beta / 2} f\right\|_{p} \leq
\end{aligned}
$$




$$
\leq C\left[t^{-\eta /(2 p)-D /(2 p)}\|f\|_{p}+t^{\beta / 2-\eta /(2 p)-D /(2 p)}\left\|A_{p}^{\beta / 2} f\right\|_{p}\right] .
$$

By choosing $t=\|f\|_{p}^{2 / \beta}\left\|A_{p}^{\beta / 2} f\right\|_{p}^{-2 / \beta}$ we obtain (4).

If $\beta>2$, we iterate (8) $n$-times and write for $n \geq \beta / 2$

$$
\begin{aligned}
f=e^{-t A} f+ & t A_{p} e^{-t A} f+\cdots+\frac{t^{n-1}}{(n-1) !} A_{p}^{n-1} e^{-t A} f \\
& +\frac{1}{(n-1) !} \int_{0}^{t} s^{n-1} e^{-(s / 2) A} A_{p}^{n-\beta / 2} e^{-(s / 2) A} A^{\beta / 2} f d s .
\end{aligned}
$$

Arguing as before, we obtain the desired conclusion.

Remark. One can also characterize the Hölder continuity of $p_{t}(x, y) e^{-t}$ by the boundedness of the operator $\left(I+A_{p}^{\beta / 2}\right)$ from some $L^{p}(X)$ into $C^{\eta}(X)$. This is easy to do but this characterization is less sharp than the previous one given in the theorem.

3. Application to multiplicative perturbations. Several applications of the result above can be given. We give here an application to mutiplicative perturbations.

Let $-A$ be the generator of a bounded holomorphic semigroup $e^{-t A}$ on $L^{2}(X)$. We assume as above that $e^{-t A}$ is uniformly bounded on $L^{p}(X), 1 \leq p \leq \infty$. We also assume that the kernel $p_{t}(x, y)$ of $e^{-t A}$ satisfies (2).

We consider now an operator $B$ which will be assumed bounded on some $L^{p}(X)$ and which satisfies the following assumption:

(H) The operator $-B A$ generates a bounded analytic semigroup $e^{-t B A}$ on $L^{2}(X)$ which is uniformly bounded on $L^{p}(X), 1 \leq p \leq \infty$.

(See [DO] for some sufficient conditions implying this assumption.) We would like to know under which reasonable conditions on $B$, the Hölder continuity estimate (1) carries over from $p_{t}(x, y)$ to the kernel of $e^{-t B A}$. It turns out that merely the boundedness of $B$ and its inverse $B^{-1}$ on some $L^{p}(X)$ is sufficient to get the estimate (1) for the kernel of $e^{-t B A}$. More precisely:

Theorem 2 Suppose that $A$ satisfies (1) and (2) with some $\eta$ and D. Suppose that $B, B^{-1} \in \mathcal{L}\left(L^{p}(X)\right)$ for some $p>\eta / 2+D / 2$ and $B$ satisfies $(\mathbf{H})$. Then $e^{-t B A}$ satisfies (2) and its kernel $k_{t}(x, y)$ satisfies (1) with $\eta / p$ in place of $\eta$.

Proof. We first show that $e^{-t B A}$ satisfies (2). By assumption the semigroup $e^{-t A}$ satisfies (2). Now by Theorem 1 of [Co1] mentionned previously, the operator $A$ satisfies the Gagliardo-Nirenberg inequality

$$
\|f\|_{\infty} \leq C\|f\|_{p}^{1-D /(2 p)}\|A f\|_{p}^{D /(2 p)}, \quad \text { for } 2 p>D
$$


Let now $p>\eta / 2+D / 2$. Since $B, B^{-1} \in \mathcal{L}\left(L^{p}(X)\right)$ we have

$$
\|A f\|_{p}=\left\|B^{-1} B A f\right\|_{p} \leq\left\|B^{-1}\right\|_{\mathcal{L}\left(L^{p}(X)\right)} \cdot\|B A f\|_{p} .
$$

Hence the operator $B A$ satisfies the estimate (9). By applying this inequality to $e^{-t B A} f$ and using the analyticity of $e^{-t B A}$ (assumption $(\mathrm{H})$ ), one easily obtains the $L^{p}-L^{\infty}$ estimate $\left\|e^{-t B A}\right\|_{p, \infty} \leq C t^{-D /(2 p)}$. Now an extrapolation result from $[\mathrm{Co} 2]$ asserts that this estimate together with assumption (H) imply (2). In particular, $e^{-t B A}$ has a kernel $k_{t}(x, y)$.

Suppose now that $p_{t}(x, y)$ satisfies (1) and apply Theorem 1 with $\beta=2$. We obtain

$$
\operatorname{Sup}_{x \neq x^{\prime}} \frac{\left|f(x)-f\left(x^{\prime}\right)\right|}{d\left(x, x^{\prime}\right)^{\eta / p}} \leq C\|f\|_{p}^{1-(1 / 2)(\eta / p+D / p)}\left\|A_{p} f\right\|_{p}^{(1 / 2)(\eta / p+D / p)} .
$$

By (10) the operator $B A$ satisfies the same inequality. We apply again Theorem 1 and deduce that $k_{t}(x, y)$ satisfies (1) with some $\eta / p$ in place of $\eta$.

\section{Application to elliptic operators. Let}

$$
A=-\sum_{j, k=1}^{D} \frac{\partial}{\partial x_{k}} a_{k j} \frac{\partial}{\partial x_{j}}
$$

be a second-order uniformly elliptic operator on $L^{2}\left(\mathbb{R}^{D}\right)$. The operator $A$ is defined as usual by sesquilinear form method. Consider a complex-valued function $b$ on $\mathbb{R}^{D}$ and assume

$$
\text { the coefficients } a_{k j} \in L^{\infty}\left(\mathbb{R}^{D}\right) \text { and are real-valued, }
$$

$$
b \in L^{\infty}\left(\mathbb{R}^{D}\right) \text { and } \Re b \geq \delta>0 \text { a.e. }
$$

where $\delta>0$ is a constant.

We assume in addition that the operator $-b A$ generates a bounded analytic semigroup $e^{-t b A}$ on $L^{2}\left(\mathbb{R}^{D}\right)$ (this holds under some sectoriality assumption on $A$ and $b$ or if $A$ is self-adjoint, see for example [DO]). We have the following result:

Theorem 3 Under the conditions above, the semigroup $e^{-t b A}$ has a kernel $k_{t}(x, y)$ and there exist positive constants $C, c, c^{\prime}, \eta \in(0,1), \eta_{0} \in(0, \eta)$ such that

$$
\begin{aligned}
\left|k_{t}(x, y)\right| & \leq C t^{-D / 2} e^{-c\left(|x-y|^{2}\right) / t} \\
\left|k_{t}(x, y)-k_{t}\left(x^{\prime}, y\right)\right| & \leq C t^{-D / 2-\eta / 2}\left|x-x^{\prime}\right|^{\eta} \\
\left|k_{t}(x, y)-k_{t}\left(x^{\prime}, y\right)\right| & \leq C t^{-D / 2-\eta_{0} / 2}\left|x-x^{\prime}\right|^{\eta_{0}} e^{-c^{\prime}\left(|x-y|^{2}\right) / t} \\
& \quad \text { for }\left|x-x^{\prime}\right| \leq \frac{1}{2}|x-y| .
\end{aligned}
$$

Moreover, the kernel $t \partial / \partial t k_{t}(x, y)$ satisfies the same three estimates. 
Proof. The Gaussian estimate (13) is shown in [DO].

The kernel $p_{t}(x, y)$ of $e^{-t A}$ satisfies (14) with some $\eta \in(0,1)$. This is the well known Aronson-Nash estimates (see [Au] or [Fr, p. 328]). The assumption (12) implies that the multiplication operators by $b$ and $b^{-1}$ are bounded on $L^{p}\left(\mathbb{R}^{n}\right)$, $1 \leq p \leq \infty$. We deduce from Theorem 2 that $k_{t}(x, y)$ satisfies (14).

In order to show (15) we write for all $\alpha \in(0,1)$

$$
\left|k_{t}(x, y)-k_{t}\left(x^{\prime}, y\right)\right| \leq\left|k_{t}(x, y)-k_{t}\left(x^{\prime}, y\right)\right|^{\alpha}\left(\left|k_{t}(x, y)\right|^{1-\alpha}+\left|k_{t}(x, y)\right|^{1-\alpha}\right)
$$

We now apply (13) and (14) and obtain (15) with $\eta_{0}=\eta \alpha$.

The kernel $t(\partial / \partial t) k_{t}(x, y)$ is nothing but the kernel of the operator $-t b A e^{-t b A}$. It is known that Gaussian bounds on the heat kernel imply Gaussian bounds on its time derivative $([\mathrm{Da} 2],[\mathrm{Gr}])$. A simple proof for non-symmetric operators is given in [DO]. One obtains then (13) for the kernel $t(\partial / \partial t) k_{t}(x, y)$ from the same estimate for $k_{t}(x, y)$. This implies in particular the $L^{1}$-estimate

$$
\left\|b A e^{-t b A} f\right\|_{1} \leq C t^{-1}\|f\|_{1} .
$$

In order to show (14), let $f \in L^{1}\left(\mathbb{R}^{D}\right)$ and $t>0$ and write $t b A e^{-2 t b A} f=$ $e^{-t b A} t b A e^{-t b A} f$. Apply now (14) for $k_{t}(x, y)$ to obtain

$$
\left|t b A e^{-2 t b A} f(x)-t b A e^{-2 t b A} f\left(x^{\prime}\right)\right| \leq C t^{-D / 2-\eta / 2}\left|x-x^{\prime}\right|^{\eta}\left\|t b A e^{-t b A} f\right\|_{1} .
$$

Using (16) we obtain the desired conclusion.

The estimate $(15)$ for $t(\partial / \partial t) k_{t}(x, y)$ can be shown in the same way as for the kernel $k_{t}(x, y)$.

The above theorem is also true if $A$ is the Dirichlet Laplacian on a smooth domain of $\mathbb{R}^{D}$ and $b$ is as above. In this case, the Hölder continuity of the heat kernel follows from Agmon-Douglis-Nirenberg estimates (see for example [Br, p. 197]) and Sobolev imbeddings $W^{2, p}(\Omega) \subset C^{\eta}(\bar{\Omega})$ for appropiate $p$ and $D$ ([Ad, p. 108]).

Remark. Using the theorem above and the method in [McN], [DO, Theorem 4.1], one obtains that the operator $b A$ has a bounded holomorphic functional calculus in $L^{p}\left(\mathbb{R}^{D}\right), 1<p<\infty$. This result is shown in $[\mathrm{McN}]$ in the case where $A$ is the Laplacian and in [DO] if either $b$ is real or $a_{k j} \in W^{1, \infty}$. By Theorem 3 the latter assumption is not needed (for more details, see these two papers).

4. Gaussian lower bounds. In [DO] it is shown that if the heat kernel $p_{t}(x, y)$ of an operator $A$ satisfies the Gaussian upper bound

$$
0 \leq p_{t}(x, y) \leq C t^{-D / 2} e^{-c\left(d(x, y)^{2}\right) / t}, \quad t>0
$$

and if $b \in L^{\infty}(X)$ and satisfies

$$
\Re b(x) \geq \delta>0 \quad \mu \text {-a.e. }
$$


then the kernel $k_{t}(x, y)$ of $e^{-t b A}$ satisfies

$$
\left|k_{t}(x, y)\right| \leq C t^{-D / 2} e^{-c\left(d(x, y)^{2}\right) / t}, \quad t>0
$$

In this section we study Gaussian lower bounds. We assume that $p_{t}(x, y)$ has a Gaussian lower bound and show (under precise assumptions) that $k_{t}(x, y)$ has the same lower bound.

Gaussian lower bounds are usually obtained from uniform on-diagonal upper and lower bounds and the Hölder continuity of the heat kernel. For the perturbed operator $b A$ we already know by Theorem 2 that we can obtain both on-diagonal upper bound and the Hölder continuity of the heat kernel. Thus we need a uniform lower bound on $k_{t}(x, x)$. More precisely, we need the estimate (19) below for $k_{t}(x, x)$. Our purpose here is to show that if $p_{t}(x, x)$ satisfies (19) then the same estimate holds for $k_{t}(x, x)$.

For symmetric heat kernels $p_{t}(x, y)$ satisfying the conservativeness property

$$
\int p_{t}(x, y) d \mu(y)=1 \text { for } \mu \text {-a.e., } x \in X,
$$

it is possible to obtain (19) from a uniform upper bound on $p_{t}(x, y)$ (under some geometrical assumptions, see [CG]). Note that in order to achieve this, both symmetry and conservativeness assumptions play an important role (the heat kernel $p_{t}(x, y)$ of the Dirichlet Laplacian on a bounded domain $\Omega$ satisfies the Gaussian upper bound but $p_{t}(x, y) \rightarrow 0$ as $\left.x, y \rightarrow \partial \Omega\right)$. Here we do not assume the conservativeness assumption in order to show (19) for the kernel $k_{t}(x, y)$ since we will start with $p_{t}(x, y)$ which satisfies (19).

From now on we assume that $A$ is self-adjoint and non-negative on $L^{2}(X)$ and denote by $e^{-t A}$ its associated semigroup. We assume that the kernel $p_{t}(x, y)$ of $e^{-t A}$ satisfies

$$
C^{\prime} t^{-D / 2} \leq p_{t}(x, x), \quad t>0 .
$$

Let now $b$ be a real-valued function on $\mathrm{X}$ which satisfies

$$
b \in L^{\infty}(X) \text { and } b(x) \geq \delta>0 \quad \mu \text {-a.e. }
$$

The operator $-b A$ with domain $D(b A)=D(A)$ generates a bounded analytic semigroup $e^{-t b A}$ on $L^{2}(X)$ (see $[\mathrm{DO}]$ or $[\mathrm{McN}]$ ). In order to apply Theorem 2 we need to extend the semigroup $e^{-t b A}$ to $L^{p}(X), 1 \leq p \leq \infty$. But this follows from (18) if for example $X$ satisfies

$$
\int_{X} t^{-D / 2} e^{-c\left(d(x, y)^{2} / t\right)} d \mu(y) \leq k, \quad \text { for a.e. } x \in X
$$

for some constant $k$ independent of $t$.

Note that the above assumption is satisfied if for example the volume of balls of radius $t$ is proportional to $t^{D}$ (see [DR, Section 2]). We also mention that since 
$b$ is real then if $e^{-t A}$ is sub-Markovian then so is $e^{-t b A}$ (see [Ou1]). This gives that $e^{-t b A}$ is uniformly bounded on $L^{p}(X), 2 \leq p \leq \infty$ without any geometrical assumption on $X$.

The following result shows that the kernel $k_{t}(x, y)$ of $e^{-t b A}$ satisfies (19).

Proposition 4 Suppose that the symmetric kernel $p_{t}(x, y)$ satisfies (17) and (19) and Suppose that $b$ satisfies (20). Then $k_{t}(x, y)$ satisfies (19).

Proof. Observe first that $e^{-t b A}=\sqrt{b} e^{-t \sqrt{b} A \sqrt{b}}(1 / \sqrt{b})$. Because of (20) it suffices to show (19) for the symmetric kernel $h_{t}(x, y)$ of $e^{-t \sqrt{b} A \sqrt{b}}$. The kernel $h_{t}(x, y)$ exists and satisfies (18) (cf. [DO]). In particular,

$$
\left|h_{t}(x, y)\right| \leq C t^{-D / 2} .
$$

Let now $\lambda>0$ and $0 \leq f \in L^{2}(X)$. We have

$$
\begin{aligned}
(\lambda I+\sqrt{b} A \sqrt{b})^{-1} f & =\frac{1}{\sqrt{b}}\left(\frac{\lambda}{b}+A\right)^{-1} \frac{1}{\sqrt{b}} f \\
& \geq \frac{1}{\|b\|_{\infty}}\left(\frac{\lambda}{\delta} I+A\right)^{-1} f .
\end{aligned}
$$

As explained in $[\mathrm{DO}]$ the above inequality can be shown easily by the Trotter product formula. Iterating $m$-times this pointwise inequality yields

$$
(\lambda I+\sqrt{b} A \sqrt{b})^{-m} f \geq \frac{1}{\|b\|_{\infty}^{m}}\left(\frac{\lambda}{\delta} I+A\right)^{-m} f .
$$

For $m>D / 2$ denote by $R_{\lambda, m}(x, y)$ the kernel of $(\lambda I+\sqrt{b} A \sqrt{b})^{-m}$. From the formula

$$
(\lambda I+A)^{-m}=\frac{1}{m !} \int_{0}^{\infty} t^{m-1} e^{-\lambda t} e^{-t A} d t
$$

and (23) we have

$$
m ! R_{\lambda, m}(x, y) \geq \frac{1}{\|b\|_{\infty}^{m}} \int_{0}^{\infty} t^{m-1} e^{-\lambda \delta^{-1} t} p_{t}(x, y) d t .
$$

Using (19), simple calculations give

$$
m ! R_{\lambda, m}(x, x) \geq C^{\prime} \frac{\delta^{m-D / 2}}{\|b\|_{\infty}^{m}} \lambda^{-m+D / 2} \Gamma(m-1-D / 2) .
$$

Let

$$
C_{m}=C^{\prime} \frac{\delta^{m-D / 2}}{\|b\|_{\infty}^{m}} \Gamma(m-1-D / 2) .
$$


We have shown

$$
C_{m} \lambda^{-m+D / 2} \leq m ! R_{\lambda, m}(x, x)=\int_{0}^{\infty} t^{m-1} e^{-\lambda t} h_{t}(x, x) d t .
$$

Using the semigroup property and the symmetry of $h_{t}(x, y)$ one obtains that $h_{2 t}(x, x)=\left\|h_{t}(x, \cdot)\right\|_{2}^{2}$. In addition, the non-negativity of $\sqrt{b} A \sqrt{b}$ implies that

$$
\frac{d}{d t} h_{2 t}(x, x)=-\left((\sqrt{b} A \sqrt{b}) h_{t}(x, \cdot), h_{t}(x, \cdot)\right) \leq 0
$$

which means that $h_{t}(x, x)$ is a decreasing function of $t$ for each fixed $x$. Hence

$$
\begin{aligned}
\int_{0}^{\infty} t^{m-1} e^{-\lambda t} h_{t}(x, x) d t \leq \int_{0}^{\lambda^{-1}} & t^{m-1} e^{-\lambda t} h_{t}(x, x) d t \\
& +h_{\lambda^{-1}}(x, x) \int_{\lambda^{-1}}^{\infty} t^{m-1} e^{-\lambda t} d t .
\end{aligned}
$$

Simple calculations give

$$
\int_{\lambda^{-1}}^{\infty} t^{m-1} e^{-\lambda t} d t=\lambda^{-m} e^{-1}(1+(m-1)+\ldots+(m-1) !)=\lambda^{-m} \alpha_{m} .
$$

Using (22), the term $\int_{0}^{1 / \lambda} t^{m-1} e^{-\lambda t} h_{t}(x, x) d t$ can be estimated by

$$
\frac{C}{m-D / 2} \lambda^{-m+D / 2} \text {. }
$$

Now (25) and these estimates give

$$
C_{m} \lambda^{-m+D / 2} \leq \lambda^{-m} \alpha_{m} h_{\lambda^{-1}}(x, x)+\frac{C}{m-D / 2} \lambda^{-m+D / 2} .
$$

Hence

$$
h_{t}(x, x) \geq \frac{1}{\alpha_{m}}\left(C_{m}-\frac{C}{m-D / 2}\right) t^{-D / 2}
$$

We choose now $m$ such that $C_{m}>C /(m-D / 2)$.

We can now deduce a Gaussian lower bound for $k_{t}(x, y)$ from the above proposition and some additionnal assumptions on $X$. The proof is classical (see [Da1, Chap. 3] or [Sc1]) but for the sake of completeness and for a unification of the arguments we will write the details.

We need the following natural assumptions on $X$.

$$
\mu(B(x, r)) \geq c r^{D} \quad \text { for all } x \in X, r>0,
$$

where $c>0$ is a constant and $B(x, r):=\{y \in X, d(x, y)<r\}$.

$$
\begin{aligned}
& \text { For } x, y \in X, n \in \mathbb{N} \text {, there exist } x_{0}=x, \\
& x_{1}, \ldots, x_{n}=y \text { such that } d\left(x_{i}, x_{i+1}\right)=\frac{1}{n} d(x, y) .
\end{aligned}
$$

We have the following result: 
Corollary 5 Suppose that $X$ satisfies (21), (26) and (27). Suppose that the symmetric kernel $p_{t}(x, y)$ satisfies $(1)$ with some $\eta \in(0,1)$ and satisfies $(17)$ and (19). Then $k_{t}(x, y)$ has a Gaussian lower bound, i.e.

$$
k_{t}(x, y) \geq M t^{-D / 2} e^{-c^{\prime}\left(d(x, y)^{2}\right) / t}, \quad t>0
$$

$\left(M, c^{\prime}\right.$ are positive constants).

Proof. We will follow [Da1, Chap. 3]. From [DO] we know that the kernel $k_{t}(x, y)$ satisfies (18). The assumption (21) implies that the semigroup $e^{-t b A}$ is uniformly bounded on $L^{p}(X), 1 \leq p \leq \infty$. We can then apply Theorem 2 and conclude that $k_{t}(x, y)$ satisfies (1) with some $\alpha \in(0,1)$. By the last proposition, $k_{t}(x, y)$ satisfies (19). So we can write

$$
\begin{gathered}
C_{1} t^{-D / 2} \leq k_{t}(x, x) \leq C t^{-D / 2}, \\
\left|k_{t}(x, y)-k_{t}(y, y)\right| \leq C d(x, y)^{\alpha} t^{-D / 2-\alpha / 2} .
\end{gathered}
$$

Consequently,

$$
k_{t}(x, y) \geq C_{1} t^{-D / 2}-C d(x, y)^{\alpha} t^{-D / 2-\alpha / 2}
$$

and hence there exist constants $\beta, C_{2}>0$ such that

$$
k_{t}(x, y) \geq C_{2} t^{-D / 2} \text { for } x, y \text { such that } d(x, y) \leq \beta \sqrt{t} .
$$

Fix now $t>0, x, y \in X$ with $d(x, y)>\beta \sqrt{t}$. By the semigroup property, for any integer $n \geq 2$

$$
k_{t}(x, y)=\int_{X^{n-1}} k_{t / n}\left(x, y_{1}\right) k_{t / n}\left(y_{1}, y_{2}\right) \ldots k_{t / n}\left(y_{n-1}, y\right) d \mu\left(y_{1}\right) \ldots d \mu\left(y_{n-1}\right) .
$$

Let $n$ be such that

$$
n-1<4 \frac{d(x, y)^{2}}{\beta^{2} t} \leq n
$$

and let $x_{0}=x, x_{1}, \ldots, x_{n}=y \in X$ be such that $d\left(x_{i}, x_{i+1}\right) \leq \beta / 2 \sqrt{t / n}$ (this is possible because of (27)). Let now $U=\left\{\left(y_{1}, \ldots, y_{n-1}\right) \in X^{n-1}, d\left(x_{i}, y_{i}\right)<\right.$ $\left.\frac{1}{4} \beta \sqrt{t / n}\right\}$. Clearly, for $\left(y_{1}, \ldots, y_{n-1}\right) \in U, y_{0}=x$ and $y_{n}=y$ we have $d\left(y_{i}, y_{i+1}\right) \leq \beta \sqrt{t / n}$ and we can then apply (30) to $k_{t / n}\left(y_{i}, y_{i+1}\right)$. So we have

$$
k_{t}(x, y) \geq \int_{U}\left(C_{2}\left(\frac{t}{n}\right)^{-D / 2}\right)^{n} d \mu\left(y_{1}\right) \cdots d \mu\left(y_{n-1}\right) .
$$

Using (26), we obtain

$$
k_{t}(x, y) \geq\left(C_{2}\left(\frac{t}{n}\right)^{-D / 2}\right)^{n}\left(c\left(\frac{1}{4} \beta \sqrt{\frac{t}{n}}\right)^{D}\right)^{n-1} .
$$


Hence $k_{t}(x, y) \geq C_{3} C_{4}^{n} t^{-D / 2}$ for some positive constants $C_{3}$ and $C_{4}$. This implies the desired conclusion (remember that $n-1<4\left(d(x, y)^{2} /\left(\beta^{2} t\right)\right) \leq n$ ).

Several examples can be given including self-adjoint elliptic operators

$$
A=-\sum_{j, k=1}^{D} \frac{\partial}{\partial x_{k}} a_{k j} \frac{\partial}{\partial x_{j}}
$$

on $L^{2}\left(\mathbb{R}^{D}\right)$. In this case the kernel $p_{t}(x, y)$ of $A$ satisfies (19) (see [Da1, Chap. $3])$. By Theorem 3 and the above corollary the heat kernel $k_{t}(x, y)$ of $b A$ has a Gaussian lower bound ( $b$ is a function satisfying (20)).

Another example is a sub-Laplacian $\Delta$ on a Lie group $G$ of polynomial volume growth (in order to have (17) we assume in addition that $d \leq D$, where $d$ and $D$ are respectively the local dimenion and the dimension at infinity). The heat kernel $p_{t}(x, y)$ satisfies (1) (this comes trivialy from the Gaussian upper bound of the space derivative shown in [Sc1]). It also satisfies (19) ([Sc1]). We then obtain that the heat kernel $k_{t}(x, y)$ of a multiplicative perturbation $b \Delta$ satisfies (1) and has both upper and lower Gaussian bounds.

Finally, the results apply also to Laplace-Beltrami operators on Riemmanian manifolds with non-negative Ricci curvature (see [LY]).

\section{REFERENCES}

[Ad] R. A. Adams, Sobolev Spaces, Academic Press, 1975

[Au $\quad$ P. Auscher, Regularity theorems and heat kernels for elliptic operators, J. London Math. Soc. 54 (1996) 284-296.

[Br] H. BrÉzis, Analyse Fonctionnelle, Theorie et Applications, Masson, Paris $3^{d}$ edition, 1992.

[Co1] Tн. Coulhon, Inégalités de Gagliardo-Nirenberg pour les semi-groupes d'opératuers et applications, Potential Anal. 1 (1992) 343-353.

[Co2] Th. Coulhon, Dimensions of continuous and discrete semigroups on $L^{p}$ spaces, in Semigroup Theory and Evolution Equations, Clément et al. (eds.), Marcel Dekker. Lectures in Pure and Appl. Math. Vol. 135 (1991), 93-99.

[Co3] Tн. Coulhon, Espaces de Lipschitz et inégalités de Poincaré, J. Funct. Anal. 136 (1996), 81-113.

[CG] Th. Coulhon And A. Grigory'An, On-diagonal lower bounds for heat kernels on non-compact manifolds and Markov chains, Duke Math. J. 89 (1997), 133-199.

[Da1] E. B. DAvies, Heat Kernels and Spectral Theory, Cambridge Univ. Press (1989).

[Da2] E. B. DAviES, Non-Gaussian aspects of heat kernel behaviour, J. London Math. Soc.(2) 5 (1997), 105-125.

[D.O] X. T. Duong And E. M. Ouhabaz, Complex multiplicative perturbations of elliptic operators: Heat kernel bounds and holomorphic functional calculus, Diff. Integ. Eq. (to appear). 
[D.R] X. T. Duong And D. W. Robinson, Semigroup kernels, Poisson bounds and holomorphic functional calculus, J. Funct. Analysis 142 (1996), 89-128.

[Fr] A. Friedman, Partial Differential Equations of Parabolic Type, Prentice Hall, New Jersey, 1964.

[Gr] A. GRIGORY'AN, upper bounds of derivatives of the heat kernel on arbitrary complete manifold, J. Funct. Anal. 127 (1995), 363-389.

$[\mathrm{McN}]$ A. McIntosh And A. NAhmod, Heat kernel estimates and functional calculi of $-b \Delta$, (Preprint 1996).

[Ou1] E. M. OuhABAZ, Semi-groupes sous-Markoviens engendrés par des opérateurs matriciels, Math. Ann. 296 (1993), 667-676.

[Ou2] E. M. Ouhabaz, Gaussian estimates and holomorphy of semigroups, Proc. Amer. Math. Soc. 123 (1995), 1465-1474.

[LY] P. Li AND S. T. YAU, On the parabolic kernel of the Schrödinger operator, Acta Math. 156 (1986), 153-201.

[Sc1] L. SAloff-Coste, Analyse sur les groupes de Lie à croissance polynomiale, Arkiv för Mat. 28 (1990), 315-331.

[Sc2] L. SAlOFF-Coste, Parabolic Harnack inequality for divergence form second order differential operators, Pot. Analysis 4 (1995), 429-467.

[VSC] N. Th. Varopoulos, L. Saloff-Coste and Th. Coulhon, Analysis and Geometry on Groups, Cambridge Univ. Press, 1992.

Equipe d'Analyse et de Mathématiques Appliquées

Université de Marne-La-Vallée

Cité Descartes

5 , boulevard Descartes, Champs-sur-Marne

F-77454 Marne-La-Vallée, cedex 2

FRANCE

EMAIL: ouhabaz@math.univ-mlv.fr

Received: February 2nd, 1998; revised: September 9th, 1998. 